\title{
IncRNA GAU1 Induces GALNT8 Overexpression and Potentiates Colorectal Cancer Progression
}

\author{
Xuemei Tang $\mathbb{D}^{1}{ }^{1}$ Haoyu Ruan $\mathbb{D}^{1},{ }^{1}$ Liu Dong $\mathbb{D}^{1},{ }^{1}$ Sihan $\mathrm{Li}^{2}{ }^{2}$ Zhiyuan Wu ${ }^{\mathbb{D}},{ }^{1}$ \\ and Ming Guan iD 1 \\ ${ }^{1}$ Department of Laboratory Medicine, Huashan Hospital, Shanghai Medical College, Fudan University, Shanghai 200040, China \\ ${ }^{2}$ Center for Pharmacogenetics, Department of Pharmaceutical Sciences, University of Pittsburgh, PA 15261, USA
}

Correspondence should be addressed to Zhiyuan Wu; wuzhiyuan126@126.com and Ming Guan; guanming88@yahoo.com

Received 14 July 2020; Accepted 4 June 2021; Published 18 June 2021

Academic Editor: Michele Manigrasso

Copyright ( 2021 Xuemei Tang et al. This is an open access article distributed under the Creative Commons Attribution License, which permits unrestricted use, distribution, and reproduction in any medium, provided the original work is properly cited.

\begin{abstract}
lncRNA is a key epigenetic regulator in biological processes. In the human cancer transcriptome library MiTranscriptome, we identified GAU1 as the top upregulated lncRNA in colorectal cancer (CRC) by sample set enrichment analysis (overexpression ranking percentile $=99.75 \%, P<10^{-50}$ ), which is coexpressed with the potential oncogene GALNT8 (Spearman rho $=0.67$, $P=2.44 \times 10^{-23}$, TCGA dataset $n=184$ ). Experimental data revealed that GAU1 regulates the expression of GALNT8. The overexpression of either GAU1 or GALNT8 significantly promotes the cell cycle and proliferation of CRC cell lines and correlates with poor prognosis in patients with CRC $\left(P=3.04 \times 10^{-2}\right)$, while silencing of GAU1 or GALNT8 suppressed the cancer cell proliferation and induced the $\mathrm{CRC}$ cell line resistance to oxaliplatin in vitro treatment. Our results suggested that the previously less studied GAU1 and GALNT8 may play as CRC prognosis markers and potential targets for chemotherapy treatment.
\end{abstract}

\section{Introduction}

Colorectal cancer (CRC) ranked the third common type of cancer, adding up $10 \%$ of all cases [1]. In 2018, there were over one million new cases and over half million deaths from the disease [2]. Genetic mutations in APC [3], TP53 [4], and $K$-RAS [5] have been intensively studied as major contributors to the tumorigenesis of CRC. Besides, nongenetic risk factors like aging and lifestyle also induce the development of CRC cases. However, this nonmutational alteration in CRC was less studied [6]. Massive parallel sequencing facilitated the genome-wide characterization of the human cancer transcriptome and identified long noncoding RNA (lncRNA) expression as the most common transcriptional alteration in cancer [7]. Our previous reports revealed that lncRNAs are extensively involved in the CRC development [8] and drug resistance [9], indicating that more efforts should be encouraged to identify the CRC-specific lncRNA expression and to link the biological "operator" regulating these noncoding "regulators."

RNA-Seq technology empowered by sequence alignment and assembly provides a revolutionary approach for the prediction of full-length transcripts from both the intergenic "gene desert" and protein-coding loci $[10$, 11]. The MiTranscriptome database applied ab initio assembly to 7,256 curated RNA-Seq libraries from tumor, normal tissue, and cell lines so as to provide an unbiased method for gene discovery [12]. Here, by incorporating this ab initio assembly-based human cancer transcriptome database and experimental validation, we identified a colorectal cancer-related lncRNA GAU1 from 12,382 cancer-associated lncRNA transcripts and verified its procancer function as upregulating the mRNA expression of polypeptide $\mathrm{N}$-acetylgalactosaminyl transferase GALNT8, whose overexpression correlates with the cancer cell proliferation and poor patient survival. 


\section{Materials and Methods}

2.1. Identification of GAU1 as the CRC-Related IncRNA. The normalized counts of $12,382 \mathrm{ab}$ initio-assembled lncRNA transcripts and library information of 6,476 RNA-Seq libraries (5,724 cancer-related samples and 752 normal samples) including 5,602 TCGA cases were downloaded from the MiTranscriptome website (http://MiTranscriptome.org/ download/MiTranscriptome.expr.counts.tsv.gz).

Sample set enrichment analysis (SSEA) [12] was performed to test if a transcript is differentially expressed between the cancer and noncancer samples in an empirical ranking method. In brief, a weighted KS test was performed as gene set enrichment analysis (GSEA) [13] to generate the enrichment score (ES) describing the enrichment of the sample set among all tested samples. SSEA was further performed 1,000 times with random permutation of the ample labels for a set of null ES and the nominal $P$ value of relative rank of observed ES within the null ES. The hypothesis testing was performed by comparing the tested ES to the null normalized enrichment score (NES) for all transcripts in a sample set. SSEA percentile score was generated by ranking the transcripts in each analysis by their NES. The tissue-type information of each transcript was obtained from the MiTranscriptome browser (http://MiTranscriptome.org).

To perform GAU1 coexpression analysis, the normalized RSEM-FPKM mRNA expression of 382 TCGA CRC samples was obtained from TCGA firehose legacy (https:/gdac .broadinstitute.org/). After sample overlapping with the MiTranscriptome database, Spearman's rank correlation coefficient of GAU1 and all 19,815 protein-coding gene mRNA expression was calculated in 184 TCGA CRC samples.

2.2. Clinical Samples and Tissue Microarray. Primary CRC tissues and paired adjacent tissues were collected from 66 CRC patients. All these samples were obtained between 2015 and 2017 and stored at $-80^{\circ} \mathrm{C}$.

Tissue microarrays (TMAs) with 55 paired cases of CRC and adjacent nontumorous tissues, plus 14 individual CRC tissues, were obtained from Shanghai Tenth Hospital (Shanghai, PR China). These CRC specimens were collected from CRC patients between 2010 and 2015 and followed until April 2019. No patient received chemotherapy or radiation before surgery, and no other concurrent cancer was observed in the patients. Both the Institutional Review Boards of Shanghai Tenth Hospital and Huashan Hospital, Fudan University, approved our study in compliance with Helsinki Declaration of 1975 as revised in 1996. All patients signed the informed consent before surgical operation. The clinical stages were classified by the American Joint Committee on Cancer and Union for International Cancer Control (AJCC/UICC) classification system [14]. Overall survival (OS) is defined as the time interval between the date of surgery and death.

2.3. Cell Culture and Stable Cell Line Establishment. Human embryonic kidney cell line HEK293T and human colon/rectum cancer cell lines LoVo, DLD1, SW620, and HCT116 were purchased from Shanghai Institute of Biological Sci- ences. All cell lines were maintained in Dulbecco's modified Eagle's medium (DMEM) (Gibco, CA, USA) with 10\% FBS (Gibco) at $37^{\circ} \mathrm{C}$ in an atmosphere of $5 \% \mathrm{CO}_{2}$.

The GALNT8 ORF sequence (NM_017417.2) and GAU1 (NR_110112.1) were cloned by reverse-transcriptional PCR from human mRNA and were further integrated into the lentiviral expression vector pCDH (Addgene, \#72265) to develop pCDH-GALNT8 and pCDH-GAU1 recombinant plasmid. Lentivirus with GALNT8 or GAU1 overexpression vector or $\mathrm{pCDH}$ control vehicle was packaged with packaging plasmid psPAX2 (Addgene, \#12260) and envelope plasmid pMD2.G (Addgene, \#12259) using Lipofectamine 2000 (Invitrogen, \#11668019) in HEK293T. Stable cell lines overexpressing GALNT8, GAU1, or vehicle control were established with SW620 and HCT116 cell lines by lentivirus infection.

2.4. siRNA Interference. siRNAs targeting GALNT8 (siGALNT8), GAU1 (siGAU1), and nonsense scramble (siNS) were purchased from Tuoran Biotech (Shanghai, China). The siRNA sequence is as follows: siGAU1-1: $5^{\prime}$-CCAAGA ACUUCGGAAGCAUTT-3' , sigAU1-2: $5^{\prime}$-CCAGCUUAC ACGUCAGCUUTT-3' , siGALNT8-1: $5^{\prime}$-CUCGAUUGU UGAAGGAAAU-3' , siGALNT8-2: $5^{\prime}$-GCUCACAGAAU GUCUACUA-3', and siNS: $5^{\prime}$-UCCTAAGGUUAAGUCG CCUC-3'. siRNA transfection of LoVo, DLD1, and their derived GAU1-overexpressing cells or vehicle control cells was undertaken with Lipofectamine RNAiMax (Invitrogen, \#13778150). All the cancer cell line transfection was performed 48 hours before further experimental usage.

2.5. Cell Proliferation and Cell Cycle Assay. Cancer cell lines were seeded $1 \times 10^{3}$ per well in the 96 -well plate. The cell proliferation was assessed by Cell Counting Kit-8 (CCK8, MCE, \#HY-K0301) every 24 hours for 5 days. The colony formation ability of cancer cell lines was measured by $0.1 \%$ crystal violet/methanol staining 10 days after cell seeding in six-well plates at $1 \times 10^{3}$ per well density. Any colony that contains more than 50 cells was counted.

Cell cycle analysis was performed with Propidium Iodide (PI) staining. A total of $10^{6}$ cells were rinsed twice with cold PBS, then fixed with $75 \%$ ethanol overnight at $-20^{\circ} \mathrm{C}$, rinsed three times with PBS, and resuspended with $0.5 \mathrm{ml} \mathrm{FxCycle}{ }^{\mathrm{TM}}$ PI/RNase Staining Solution (Life Technologies, \#F10797). Keep the cell suspension for $15 \mathrm{~min}$ in the dark, and immediately subject to flow cytometry analysis on a FACSCanto system (BD Biosciences).

2.6. Quantitative Real-Time PCR. Trizol reagent (Invitrogen) was used for total RNA of tissues or cell extraction. Reverse transcription was performed with PrimeScript ${ }^{\mathrm{TM}}$ RT Reagent Kit (TaKaRa Biotechnology, \#RR047A). Quantitative real-time PCR was conducted with TB Green Premix (TaKaRa Biotechnology, \#RR820A) and gene-specific primers (Table 1) on an Applied Biosystems 7500 system (ABI); $\beta$-actin was used as a mRNA expression housekeeping gene (Table 1). Relative expression of GALNT8 and GAU1 was calculated with the $2^{-\Delta \Delta \mathrm{Ct}}$ method. 
TABle 1: Primer sequences for gene amplification.

\begin{tabular}{lcc}
\hline Gene & Strand & Sequences $\left(5^{\prime}-3^{\prime}\right)$ \\
\hline \multirow{2}{*}{ GALNT8 } & $\begin{array}{l}\text { Forward } \\
\text { Reverse }\end{array}$ & $\begin{array}{l}\text { ACGCCCTCTCGATTGTTGAA } \\
\text { CTCTGCCCACCCAACATTGA }\end{array}$ \\
\hline \multirow{2}{*}{ GAU1 } & Forward & GCCCTTCCCAAAGCACAAAT \\
& Reverse & AGCACGTTAAGAGGCTTGGA \\
\hline \multirow{2}{*}{$\beta$-Actin } & Forward & TTGTTACAGGAAGTCCCTTGCC \\
& Reverse & ATGCTATCACCTCCCCTGTGTG \\
\hline
\end{tabular}

2.7. Antibody and Regent Information. The primary antibodies are GALNT8 (Abcam, \#ab121374) and $\beta$-actin (Cell signaling technology, \#3700). The secondary antibodies are HRP-labeled goat anti-rabbit IgG (Thermo Fisher, \#31460) and HRP-labeled goat anti-mouse IgG (Thermo Fisher, \#31430). Oxaliplatin was purchased from MCE (\#HY-17371).

2.8. Protein Isolation and Immunoblotting. Cancer cell samples were suspended with $0.05 \%$ trypsin and washed twice with cold PBS and, after, homogenized with RIPA lysis buffer and protease inhibitor cocktail (Beyotime Biotechnology, \#P0013D) on ice for 30 minutes. Cell lysates were harvested by $4^{\circ} \mathrm{C}$ centrifuge and diluted with $2 \times$ SDS sample buffer. The denaturized protein samples were resolved by SDSPAGE and transferred onto polyvinylidene fluoride (PVDF) membranes (Millipore, \#ISEQ00010). Blocked with 5\% skimmed milk in PBST, the PVDF membranes were incubated with specific primary antibodies overnight at $4^{\circ} \mathrm{C}$. After 3 times of 10-minute TBST buffer rinsing, the membranes were again incubated with secondary antibodies for 1 hour at room temperature and rinsed 3 times with TBST buffer for 10 minutes. Signals were detected with enhanced chemiluminescence (ECL) substrate (ThermoFisher, \#32106) on a Las-3000 Luminescent Image Analyzer (Fujifilm, Japan).

2.9. TMA Staining and Immunohistochemistry. The TMA slide was air-dried at $60^{\circ} \mathrm{C}$ for an hour and treated with $0.01 \mathrm{M}$ citric acid buffer solution for antigen retrieval. After cooling down to room temperature, the slide was further treated by $3 \% \mathrm{H}_{2} \mathrm{O}_{2}$ solution in methanol for 10 minutes and rinsed 3 times with cold PBS before incubation with primary anti-GALNT8 antibody $(1: 100)$ at $4^{\circ} \mathrm{C}$ overnight. The slides were rinsed three times for 5 minutes and then incubated with ready-to-use biotinylated goat anti-rabbit IgG (Abcam, \#ab64256) solution for 15 minutes at room temperature, followed by PBS rinsing for five times. Streptavidin peroxidase complex (Abcam, \#ab64269) was applied to the TMA and incubated for 10 minutes at room temperature and rinsed by PBS for five times. After visualization with diaminobenzidine chromogen (Abcam, \#ab64238) and hematoxylin counterstaining, the TMA was imagined using a Nikon Eclipse E-800 microscope. The stained TMA was then independently reviewed by two pathologists and rated for the grade of GALNT8 staining with scores of,$-+/-,+,++$, and +++ .
2.10. Cytotoxic Assay. For SW620 and DLD1, the cells with manipulated GAU1 expression or control were seeded in the 96-well plate at a density of $1 \times 10^{3}$ cells per well and incubated with low serum medium ( $1 \% v / v$ FBS) with or without oxaliplatin. Cells were replenished with fresh low serum medium with or without oxaliplatin on the third day. Cell Counting Kit-8 (CCK8, MCE, \#HY-K0301) assay was used to estimate the cell viability at the end of the fifth day of treatment.

2.11. Subcellular Isolation. Subcellular isolation in LoVo and DLD1 cells was performed as described [15] with modification. Prepare isolation buffer (1.28 M sucrose; $40 \mathrm{mM}$ Tris- $\mathrm{HCl}, \mathrm{pH}$ 7.5; $20 \mathrm{mM} \mathrm{MgCl}_{2}$; and 4\% Triton-X 100) and diluted isolation buffer $\left(\right.$ cold $\mathrm{H}_{2} \mathrm{O}$ : cold PBS : isolation buffer $=3: 1: 1$ ). $10^{6}$ cells were suspended in $200 \mu \mathrm{l}$ diluted isolation buffer and incubated on ice for $10 \mathrm{~min}$. $20 \mu \mathrm{l}$ lysate was added to $1 \mathrm{ml}$ Trizol for total RNA extraction. The rest of the lysate was rotated at $4^{\circ} \mathrm{C}$ for $20 \mathrm{~min}$ and centrifuged at $2500 \times \mathrm{g}$ for $15 \mathrm{~min}$ at $4^{\circ} \mathrm{C}$. Add $1 \mathrm{ml}$ Trizol to the supernatant for cytoplasmic RNA extraction. Wash the pellet once, resuspend with $160 \mu \mathrm{l}$ isolation buffer, and add $1 \mathrm{ml}$ Trizol for the nuclear RNA extraction. Fractionated RNAs were used for cDNA synthesis and qRT-PCR.

2.12. Statistical and Survival Analysis. GraphPad Prism 7 (La Jolla, CA, USA) and SPSS 20 (IBM, NY) were used for statistical analysis and graph preparation. All data are displayed as means \pm SD. Two-tailed Student's $t$-test was used for assessment of differences between any two groups. Kaplan-Meier analysis was used to perform survival analysis, and the patients' survival comparison between subgroups was analyzed with log-rank test. Nonparametric WilcoxonMann-Whitney test was performed for patients' clinical data analysis.

\section{Results}

3.1. Identification of GAU1 as the Colorectal Cancer-Related $\ln c R N A$. To identify the colorectal cancer-related lncRNA in the MiTranscriptome database, we first performed the differential expression analysis for all the 5,724 cancer libraries vs. 752 noncancer libraries by SSEA and annotated all the transcripts with tissue-type information (Figure 1(a)). After the empirical ranking test, two transcripts of GAU1 (ENSG00000255474) were listed on the top CRC-related lncRNA (ranking percentile $=99.75 \%$ of 12,382 ) besides our previously reported CRC-specific IncRNA PHiL [9] (ranking percentile $=99.62 \%$ ).

Then, we further experimentally quantified the GAU1 mRNA overexpression in human colorectal cancer cell lines SW620, HCT116, DLD1, and LoVo versus human intestinal epithelial cell line NCM460 $(P<0.05$, Figure 1(b)). Furthermore, qRT-PCR of GAU1 mRNA in 66 pairs of CRC tissues and adjacent normal tissues also confirmed GAU1 as the cancer-specific lncRNA in CRC $\left(P=2.53 \times 10^{-2}\right.$, Figure 1(c)). More importantly, the Kaplan-Meier analysis revealed that patients with higher GAU1 expression had worse prognosis $\left(P=3.04 \times 10^{-2}\right.$, Figure $\left.1(\mathrm{~d})\right)$, indicating GAU1 may play an oncogenic role in CRC. And the 


\section{Identification of CRC-related lncRNA in MiTranscriptome}

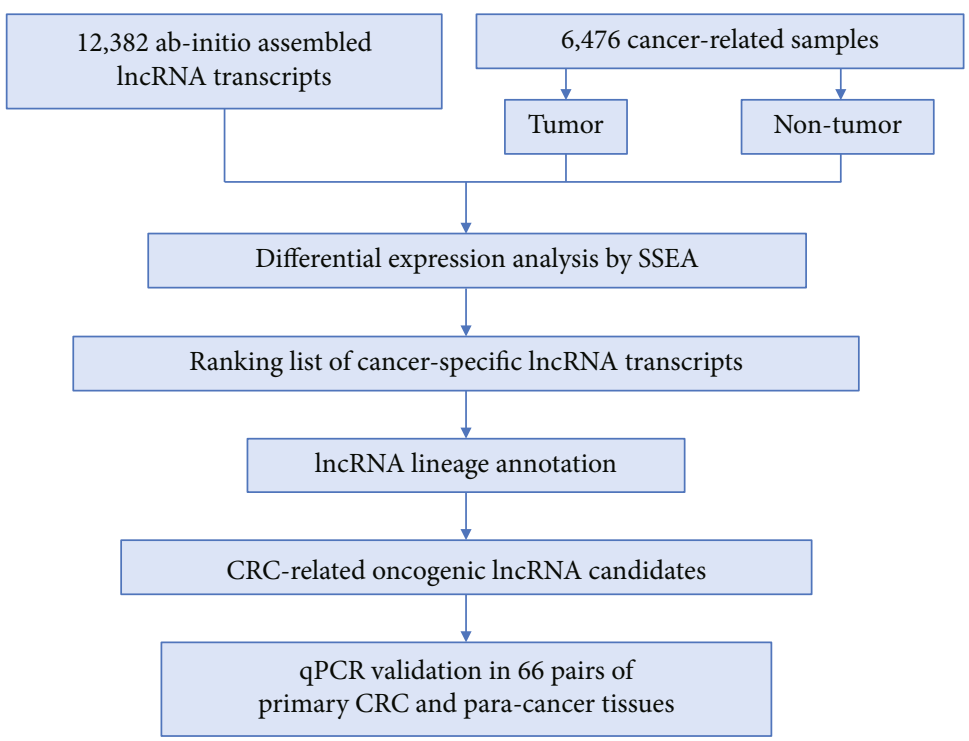

(a)
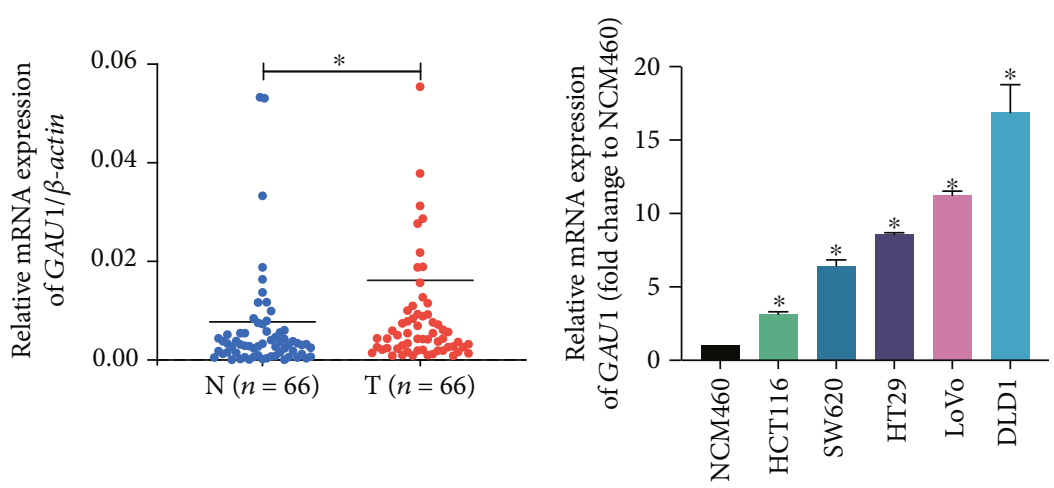

(b)

(c)

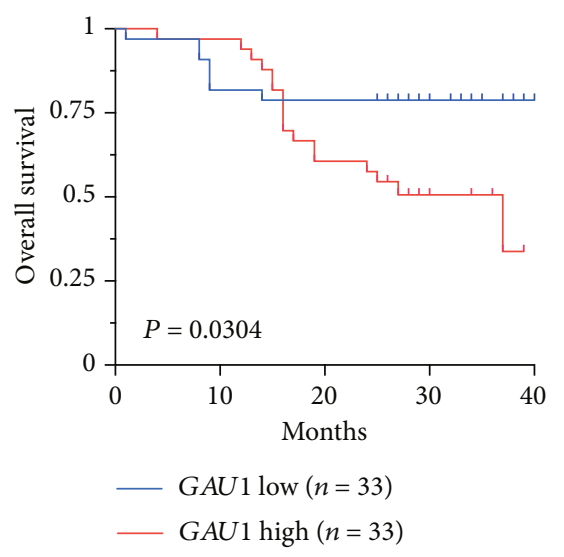

(d)

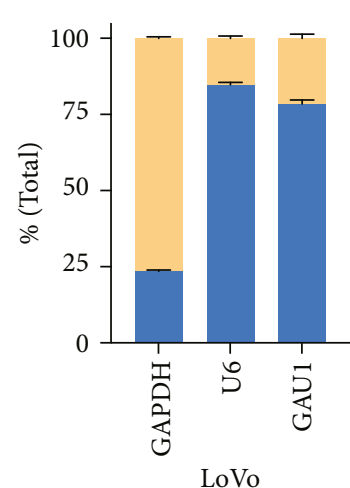

LoVo

Cytoplasm

Nucleus

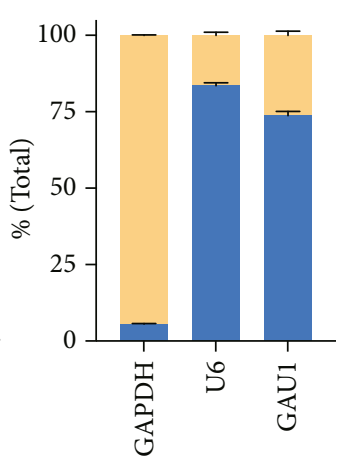

DLD1

(e)

FIGURE 1: Identification of GAU1 as the colorectal cancer-related lncRNA. (a) The working pipeline of identifying CRC-related lncRNA in the MiTranscriptome database. (b) Relative expression level of GAU1 detected by qRT-PCR in 66 paired colorectal cancer (CRC) tissues and adjacent normal tissues $\left(P=2.53 \times 10^{-2}\right)$. N: adjacent normal tissues; T: tumor tissues. (c) qRT-PCR analysis of GAU1 expression in the five CRC cell lines and the normal NCM460 cells was tested. ${ }^{*} P<0.05$. (d) Kaplan-Meier analysis of the correlation between GAU1 mRNA expression and overall survival in 66 CRC patients $\left(P=3.04 \times 10^{-2}\right)$. (e) Total RNA from LoVo and DLD1 cells was separated into cytoplasmic and nuclear fractions and analyzed by qRT-PCR. GAPDH serves as a positive control for cytoplasmic gene expression, and U6 as a positive control for nucleolus separation. 


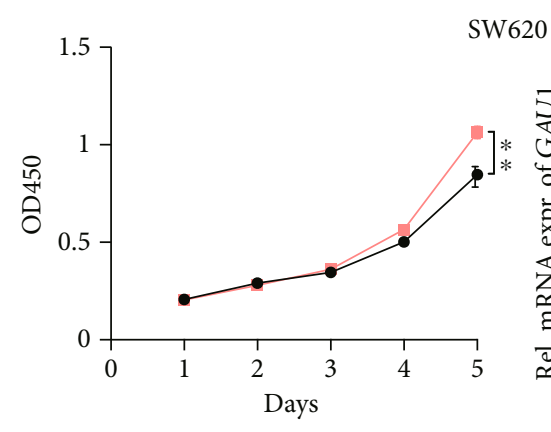

SW620

SW620
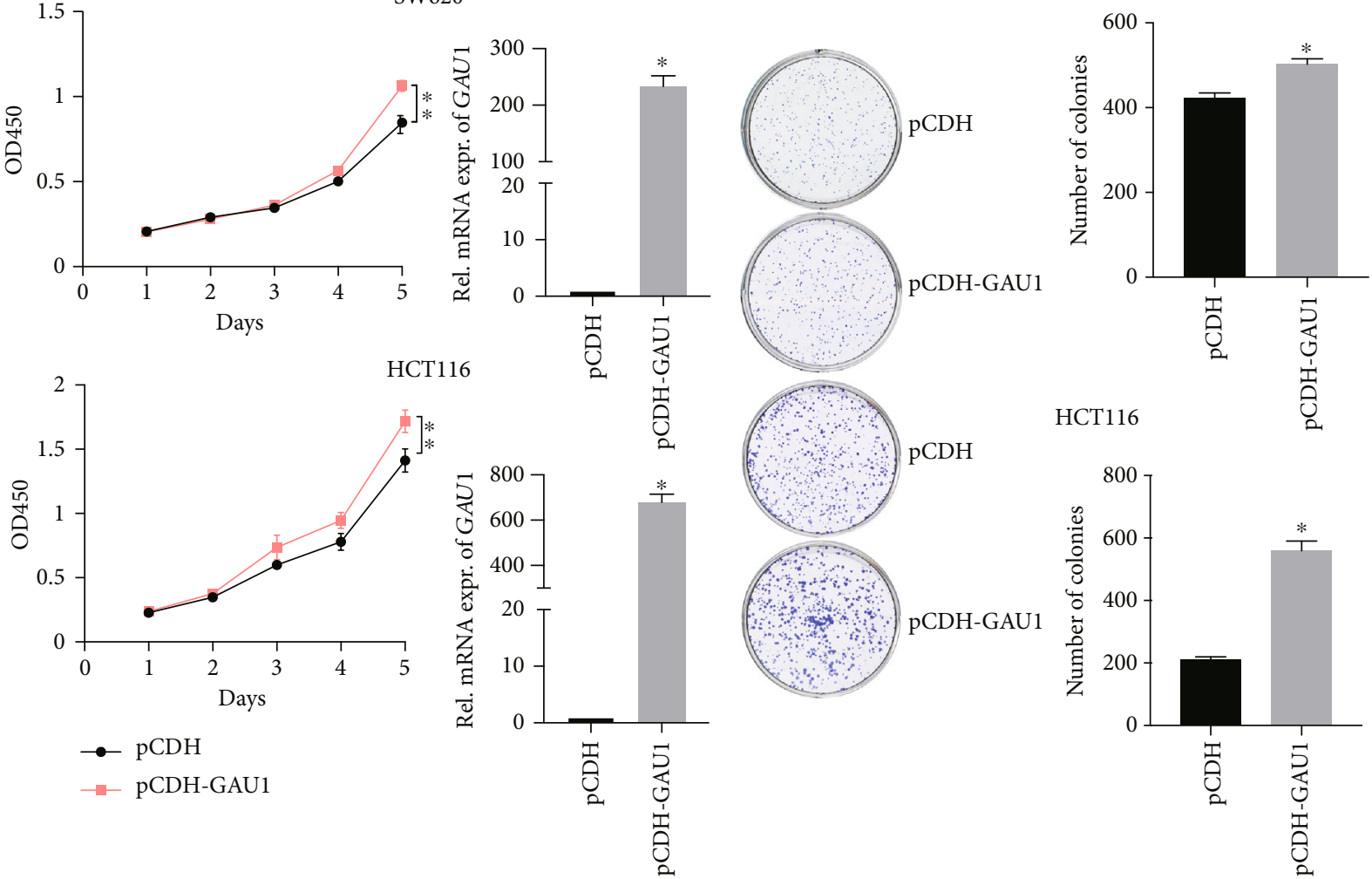

(a)

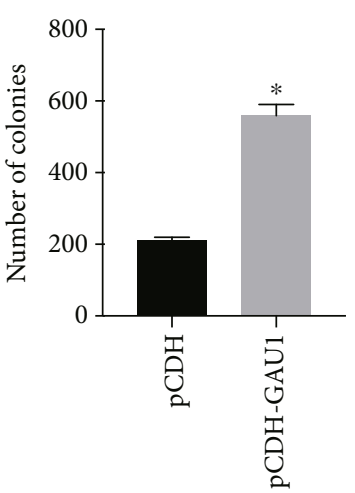

(b)
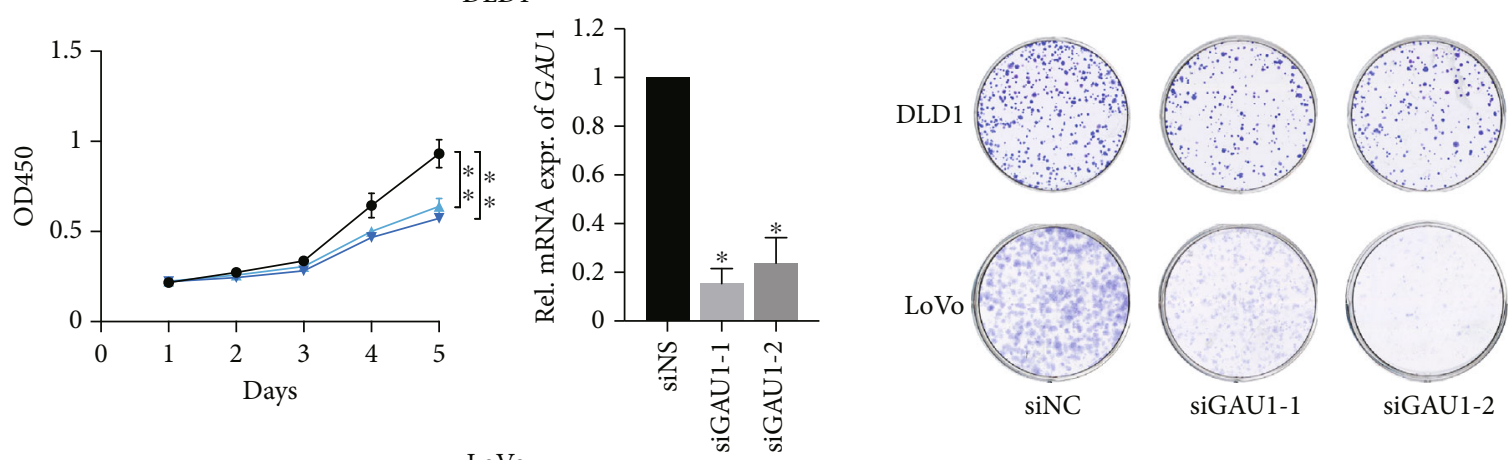

LoVo
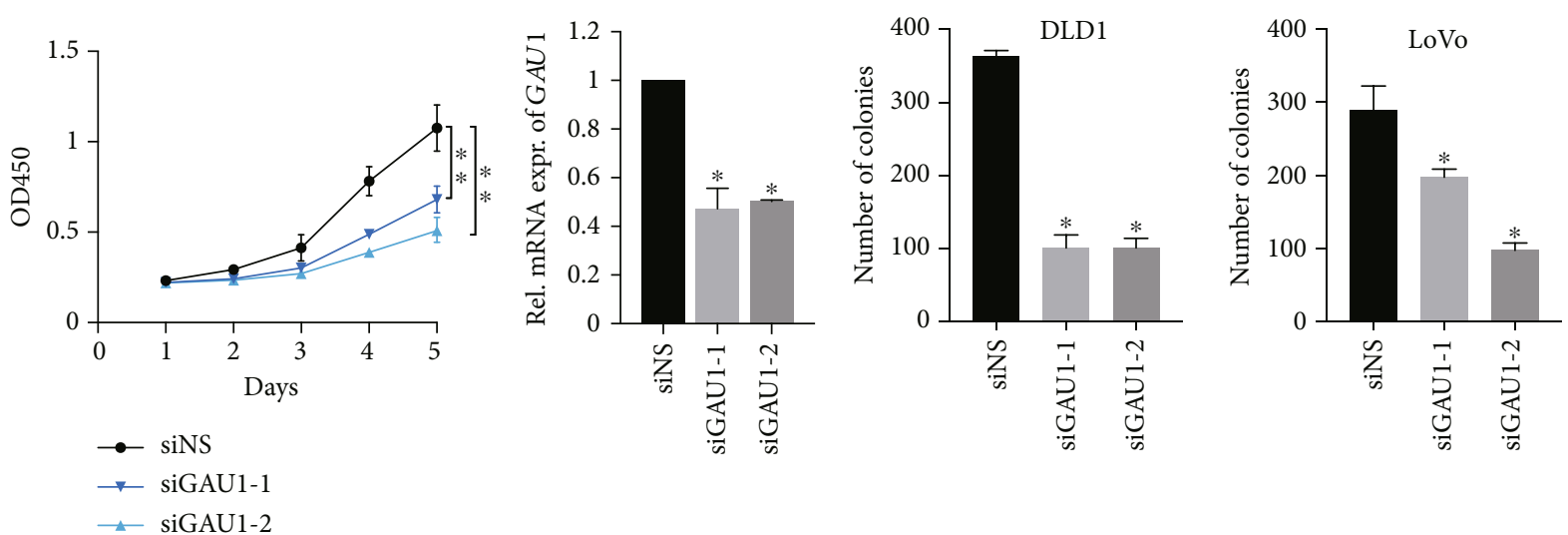

(c)

(d)

FIgUre 2: Continued. 

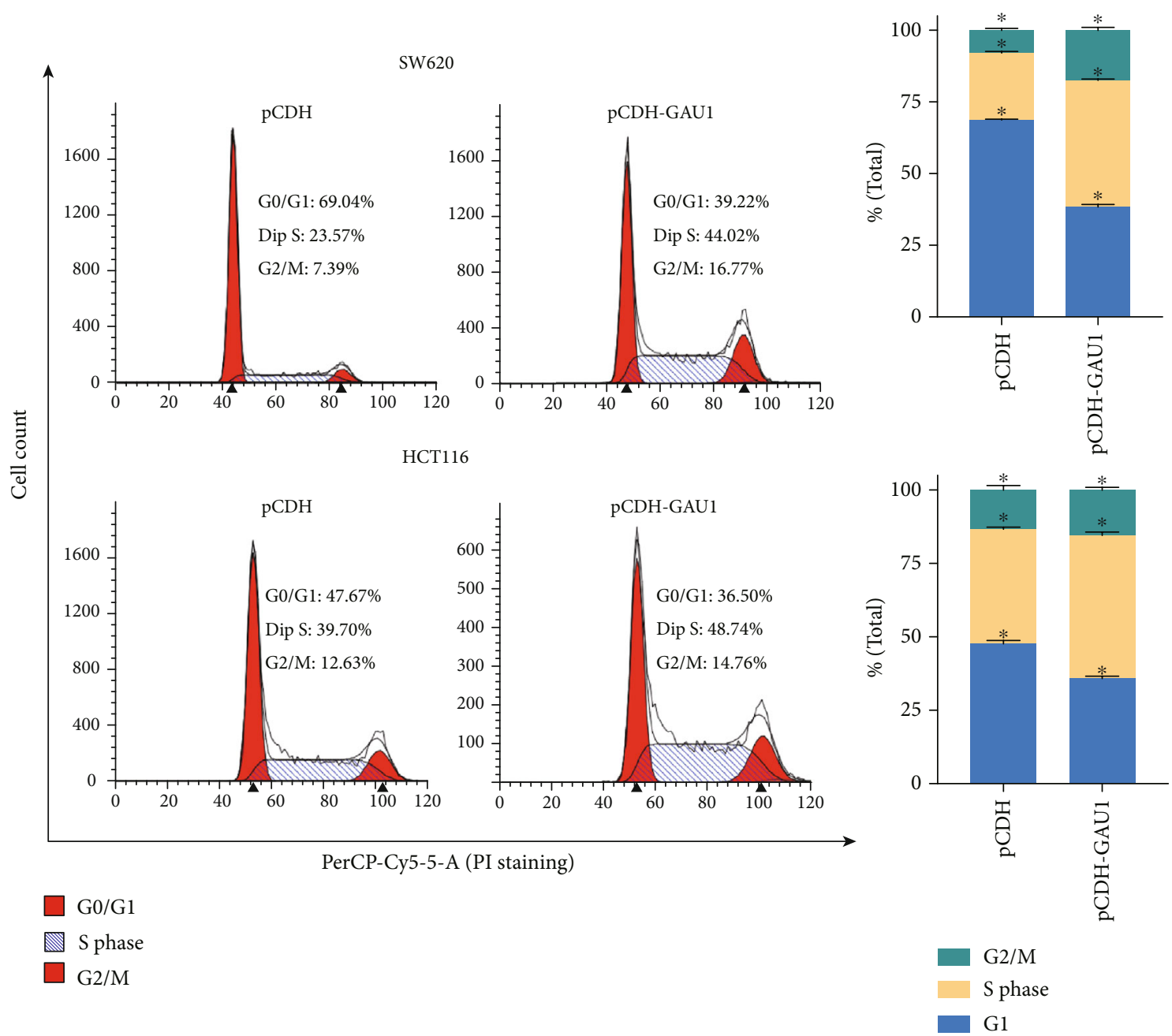

(e)

FIGURE 2: GAU1 overexpression facilitates CRC cell proliferation. (a) CCK-8 assay and (b) colony formation assay were conducted in SW620 and HCT116 cells transfected with GAU1 overexpression or control plasmids. ${ }^{*} P<0.05$; ${ }^{* *} P<0.01$. (c) CCK-8 assay and (d) colony formation assay showed the proliferation of control siRNA (siNS) or siRNAs (siGAU1-1 and siGAU1-2) against GAU1-transfected DLD1 and LoVo cells. ${ }^{*} P<0.05 ;{ }^{* *} P<0.01$. (e) Cell cycle analysis of GAU1-overexpressed HCT116, SW620, and their controls.

subcellular localization showed that GAU1 was mainly distributed in the nucleus (Figure 1(e)).

\subsection{GAU1 Overexpression Facilitates CRC Cell Proliferation} by Promoting Cell Cycle. To further determine if the GAU1 overexpression can alter the biological phenotype of CRC, we first established the GAU1-overexpressing stable cell lines by lentiviral infection of pCDH-GAU1 in SW620 and HCT116 cell lines with intermediate GAU1 expression. The CCK-8 and clonogenic assays both revealed that GAU1 overexpression lead to a significantly increased cell proliferation in the CRC cell lines compared to the vehicle controls (Figures 2(a) and 2(b)). Consistently, GAU1 knockdown in the GAU1 high-expressing LoVo and DLD1 cell lines by short interfering RNA (siRNA) significantly reduced the cell proliferation and clonogenic ability of the CRC cells (Figures 2(c) and 2(d)). These data suggested that GAU1 overexpression promotes CRC cell proliferation in vitro. Moreover, the cell cycle profile alteration after GAU1 overex- pression (increased S-phase commitment) (Figure 2(e)) also implied GAU1 as a critical player in promoting S-phase entry.

3.3. GALNT8 as the Oncogenic Operator of GAU1 in CRC. To identify the biological "operator" of GAU1 overexpression in CRC development, we performed coexpression analysis for GAU1 in 184 TCGA CRC samples. Correlation analysis revealed GALNT8, located in the vicinal gene loci of GAU1 on chromosome 12, as the most significantly coexpressed gene of GAU1 among all the 19,815 protein-coding genes (Spearman rho $=0.67, P=2.44 \times 10^{-23}$, Figure $3(\mathrm{a})$ ). The strong expression correlation between GAU1 and GALNT8 was further validated in our 66 pairs of clinical samples $\left(P<10^{-4}\right.$, Figure 3(a)), with a significant upregulation of GALNT8 expression in the tumor tissues $(\mathrm{T})$ compared with the adjacent nontumorous tissues $(\mathrm{N})\left(P<10^{-4}\right.$, Figure 3(b)). Clinically, the Kaplan-Meier analysis also revealed that patients with higher GALNT8 expression had worse overall survival $\left(P=0.31 \times 10^{-2}\right.$, Figure $\left.3(\mathrm{c})\right)$. 

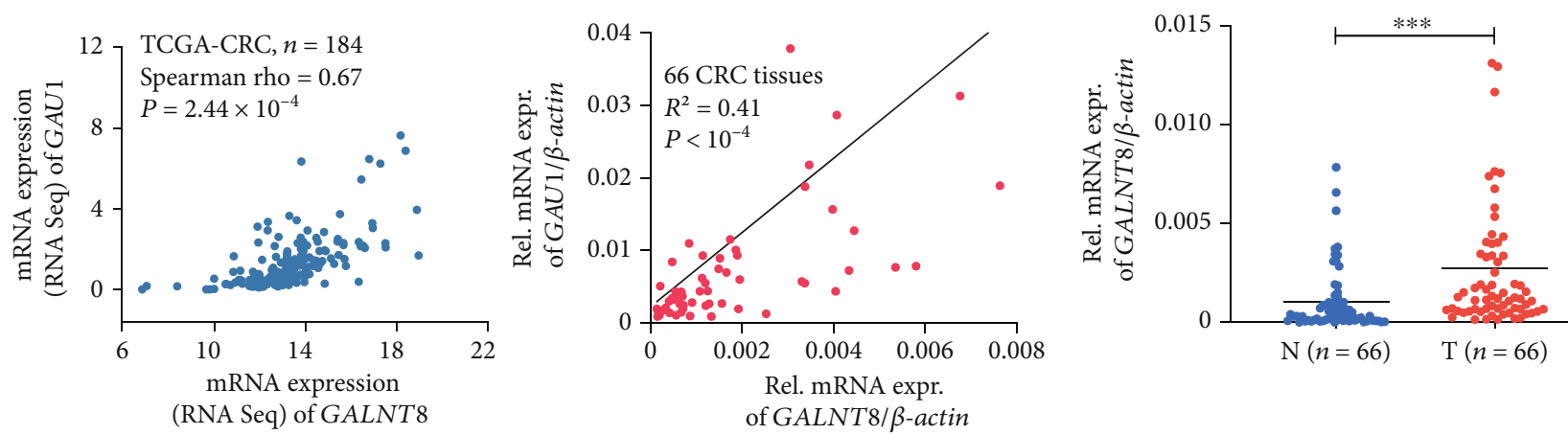

(a)

(b)
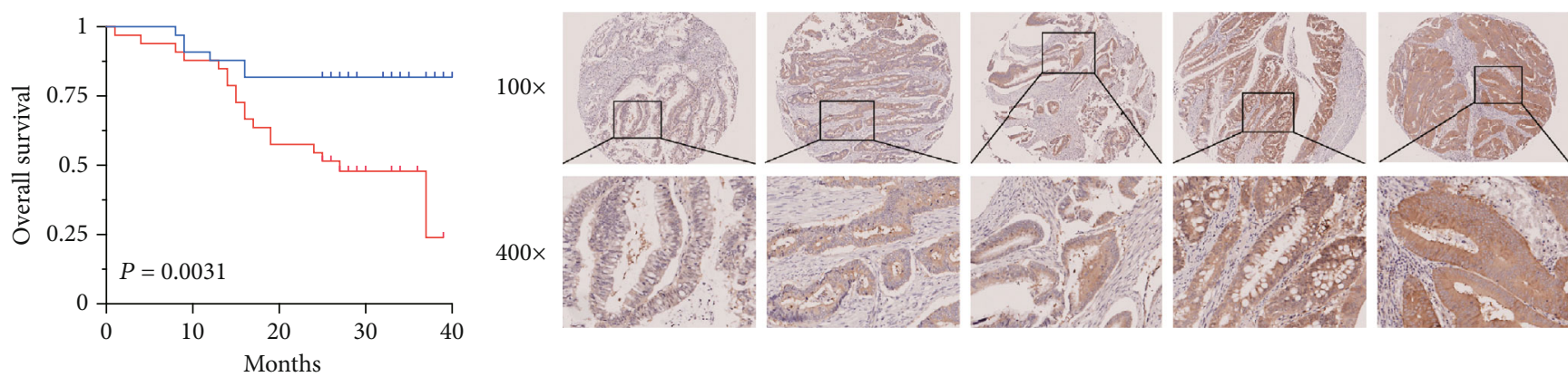

- GALNT8 low $(n=33)$

- GALNT8 high $(n=33)$

(c)

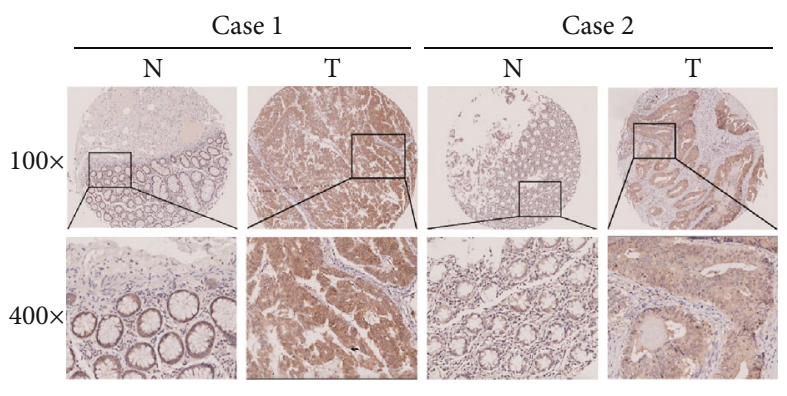

(e) (d)

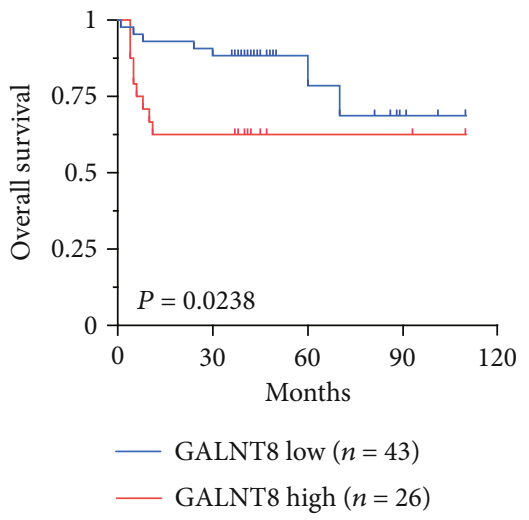

(f)

Figure 3: Continued. 


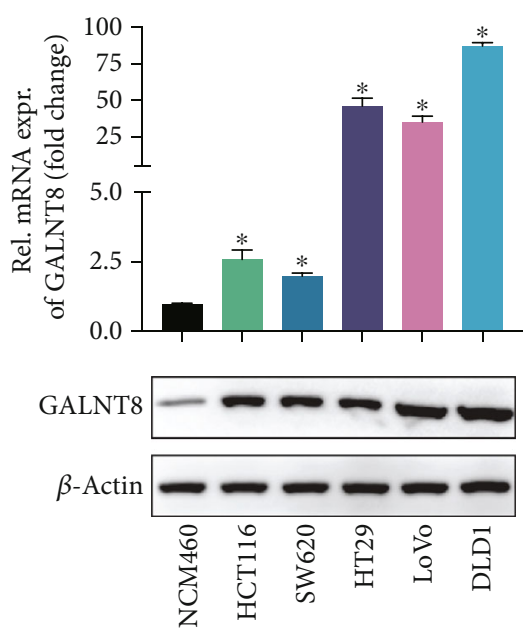

(g)
SW620

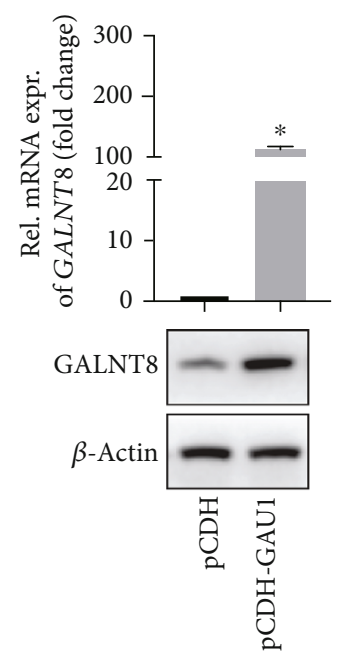

HCT116
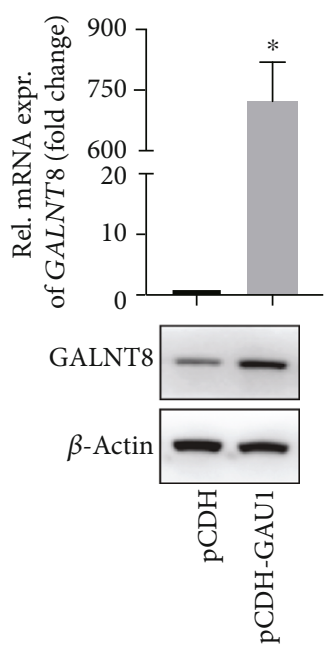

(h)
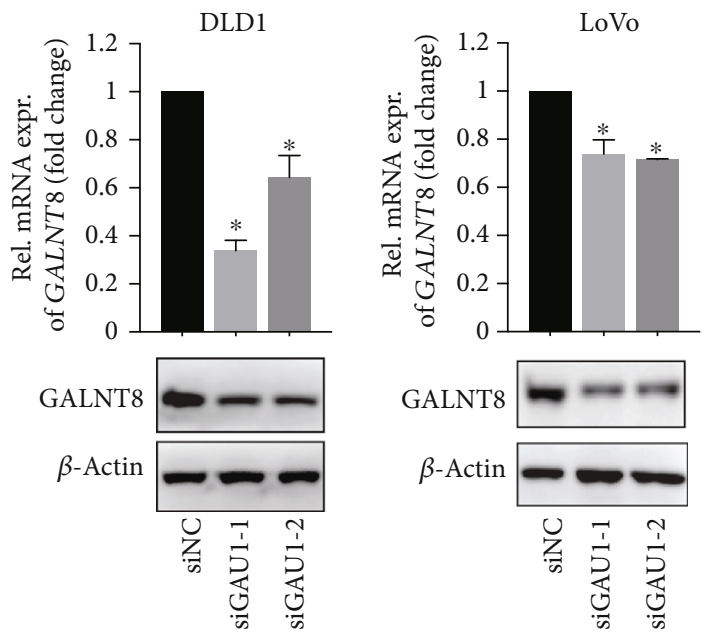

(i)

Figure 3: GALNT8 as the oncogenic operator of GAU1 in CRC. (a) Correlation analysis of GALNT8 and GAU1 in 184 TCGA CRC samples (Spearman rho $=0.67, P=2.44 \times 10^{-23}$ ), and 66 frozen CRC tissues (qRT-PCR, $R^{2}=0.41, P<10^{-4}$ ). (b) qRT-PCR analysis of GALNT8 expression level in 66 paired CRC tissues and adjacent normal tissues $\left(P<10^{-4}\right)$. N: adjacent normal tissues; T: tumor tissues. (c) KaplanMeier analysis of GALNT8 and overall survival in 66 CRC patients. (Kaplan-Meier $P=0.31 \times 10^{-2}$ ). (d) Representative photomicrographs of GALNT8 in CRC specimen TMA (magnification: $\times 100, \times 400)$. (e) Two paired tumor-adjacent control representative cases of GALNT8 expression in the TMA (magnification: $\times 100, \times 400$ ). (f) Kaplan-Meier analysis of GALNT8 expression and overall survival in 69 TMA samples. (Kaplan-Meier $P=2.38 \times 10^{-2}$ ). (g) qRT-PCR and western blot analysis of GAU1 expression in the five CRC cell lines and the normal NCM460 cells was tested. ${ }^{*} P<0.05$. (h) qRT-PCR and western blot analysis of GALNT8 expression level in GAU1-overexpressed SW620 and HCT116 cells, as well as (i) GAU1-knockdown DLD1 and LoVo cells. ${ }^{*} P<0.05$.

The overexpression of GALNT8 in the CRC patients was further validated by the IHC staining of a TMA containing 55 paired cases of CRC and adjacent nontumorous tissues, plus 14 individual CRC tumors. According to the density of IHC staining (Figure 2(d)), GALNT8 protein expression in tumor tissues was classified as high expression (score ++ , score +++ ) in 26 cases $(26 / 69,37.68 \%)$ and low expression (score + , score $+/-$, and score -) in 43 cases $(43 / 69,62.32 \%$ ) (Figure 3(d)). Tumor tissues harbored a significantly increase GALNT8 expression compared to the adjacent nontumorous tissues (Fisher exact $P=3.30 \times 10^{-8}$, Figure 3(e)). Further survival assay by Kaplan-Meier analysis also revealed that
CRC patients with overexpressed GALNT8 suffered from poor overall survival $\left(P=2.38 \times 10^{-2}\right.$, Figure 3(f)).

Moreover, in contrast to human intestinal epithelial cell line, a higher expression of GALNT8 in CRC cells was observed in both mRNA and protein levels (Figure 3(g)). To further confirm the regulatory effect of GAU1 on GALNT8 expression, the effect of GAU1 knockdown/overexpression on the expression levels of GALNT8 in CRC cells was determined. The mRNA and protein expression levels of GALNT8 were increased in the GAU1 overexpression cell lines and decreased in the siGAU1 cell lines compared with the control group (Figures 3(h) and 3(i)). Altogether, the 

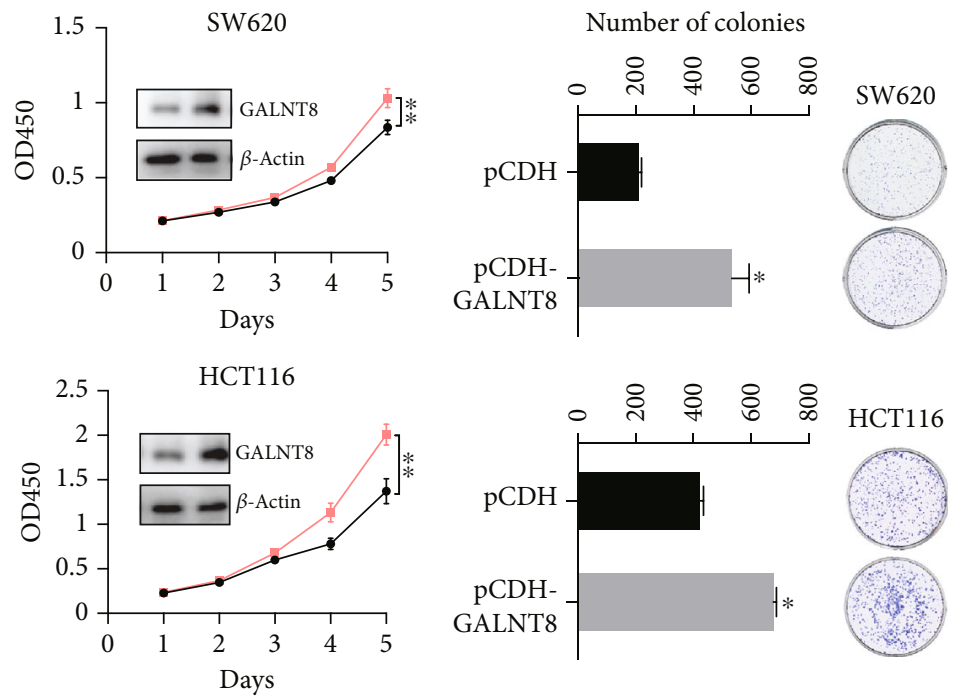

$\bullet \mathrm{pCDH}$

pCDH-GALNT8

(a)

(b)
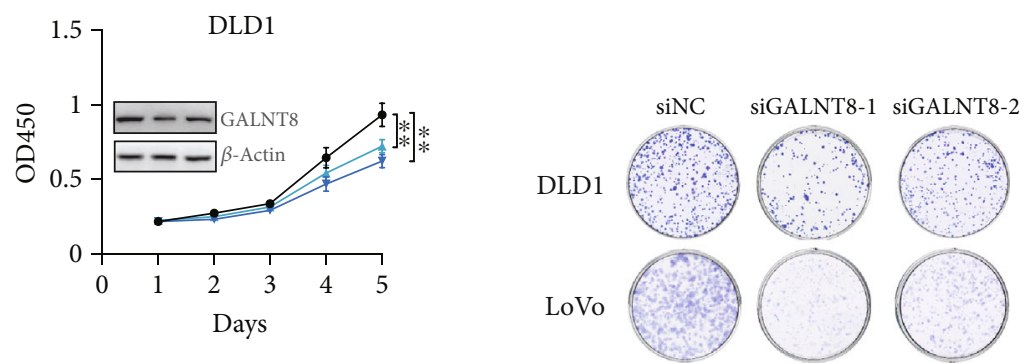

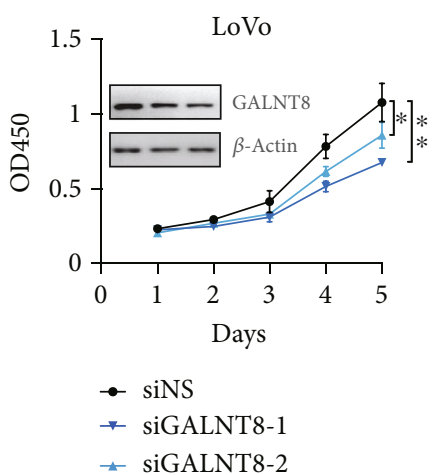

(c)
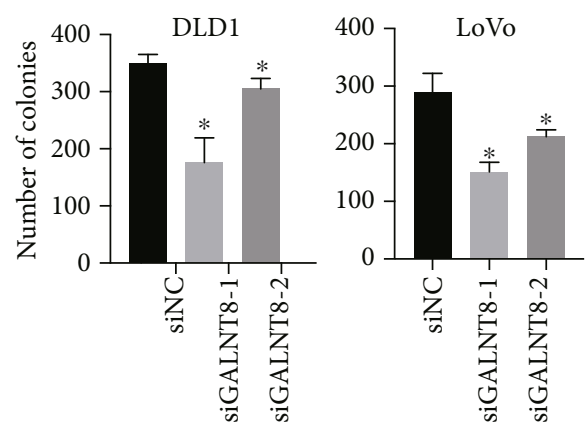

(d)

FIgURe 4: Continued. 

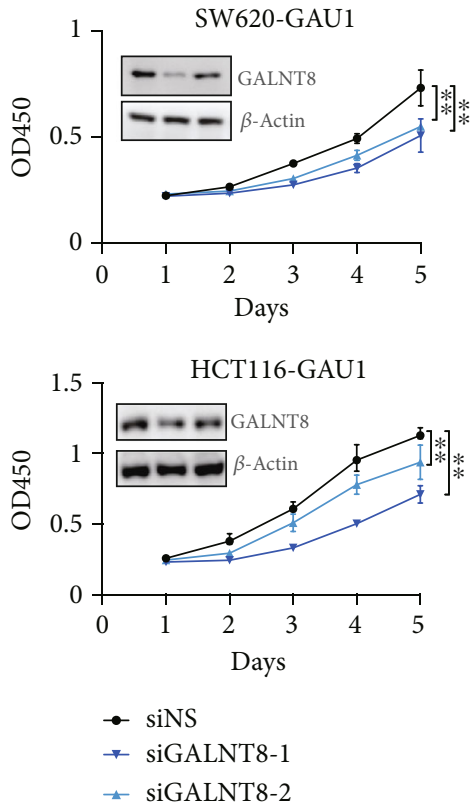

(e)
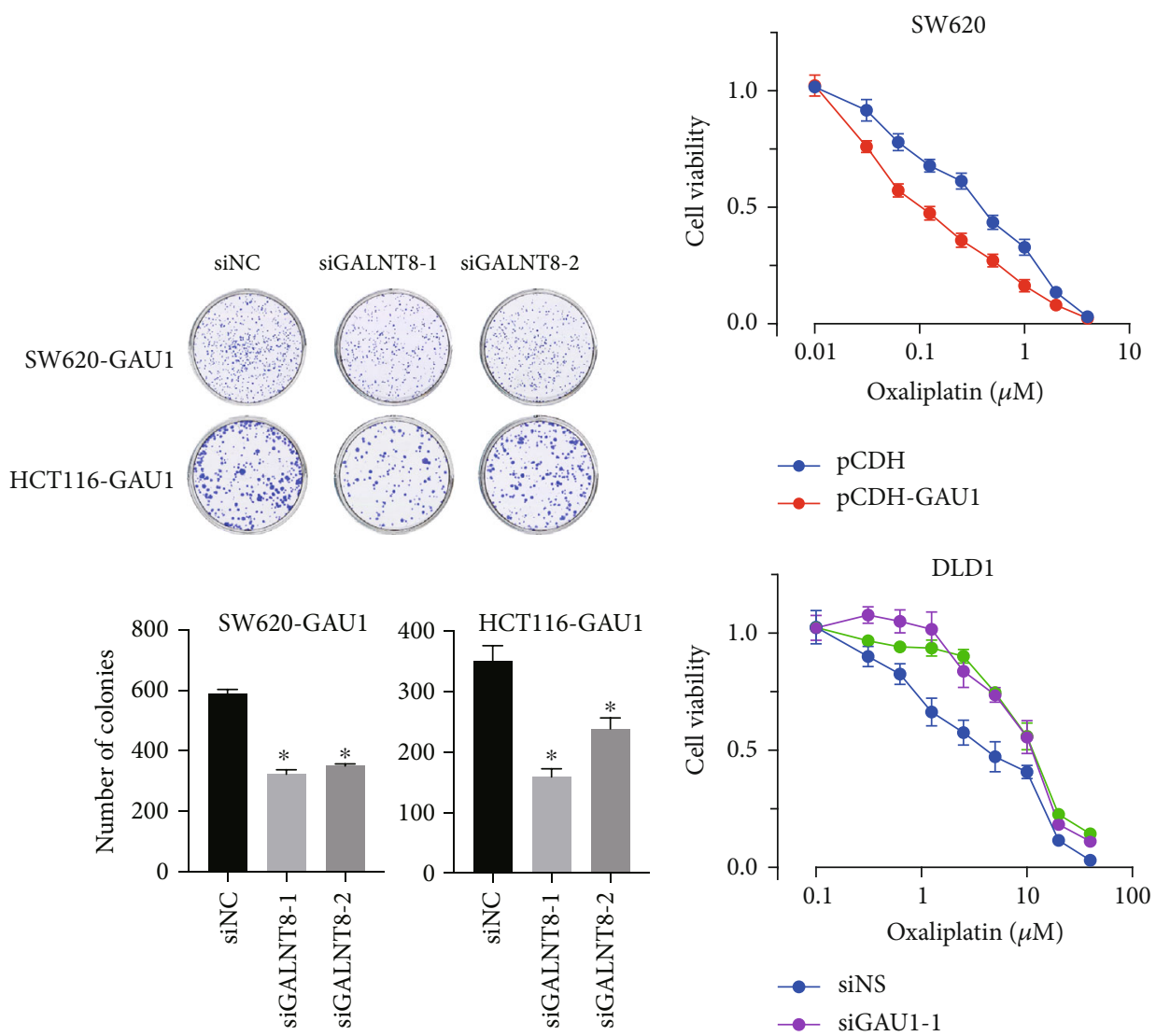

(f)

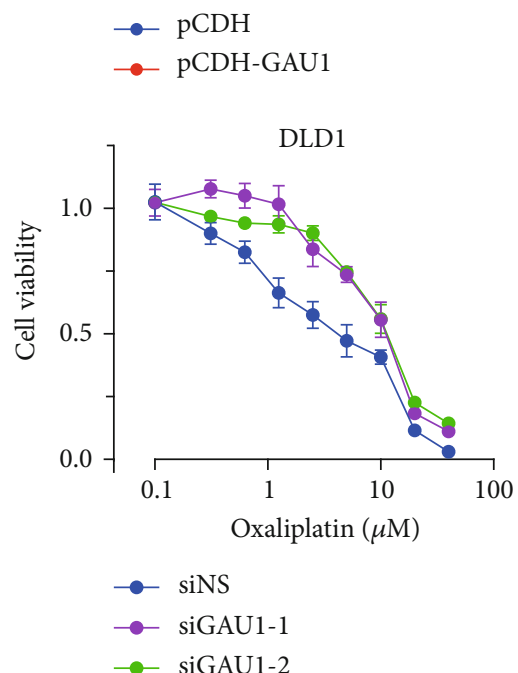

(g)

FIgURE 4: The oncogenic ability of GAU1 is GALNT8 dependent, and overexpression of GAU1/GALNT8 axis sensitizes CRC cell lines to chemotherapy. (a) CCK-8 assay and (b) colony formation assay were used to measure proliferation in SW620 and HCT116 cells transfected with pCDH-GALNT8 or pCDH. ${ }^{*} P<0.05$; ${ }^{* *} P<0.01$. (c) CCK-8 assay and (d) colony formation assay were conducted in control siRNA (siNS) or siRNAs (siGALNT8-1 and siGALNT8-2) against GALNT8-transfected DLD1 and LoVo cells. ${ }^{*} P<0.05$; ${ }^{* *} P<0.01$. (e, f) Proliferation analysis by (e) CCK-8 assay and (f) colony formation assay in GAU1-overexpressed SW620 and HCT116 cells transfected with control siRNA (siNS) or siRNAs (siGALNT8-1 and siGALNT8-2) against GALNT8. ${ }^{*} P<0.05$; ${ }^{* *} P<0.01$. (g) Viability of pCDH-GAU1- or pCDH-transfected SW620 and siGAU1-1/2- or siNS-transfected DLD1 cells incubated with multiple concentrations of oxaliplatin $(0.01-40 \mu \mathrm{M})$ was monitored through CCK-8 assay.

computational and experimental evidence suggested GALNT8 as a regulatory downstream molecule of GAU1 in CRC.

\subsection{The Oncogenic Ability of GAU1 Is GALNT8 Dependent.} Since the relationship between GALNT8 and cancer is limited, we experimentally manipulated the expression of GALNT8 by lentiviral stable overexpression and siRNA interference. CCK-8 and colony forming assays demonstrated that the overexpression of GALNT8 enhanced the proliferation and colony formation capacity of SW620 and HCT116 (Figures 4(a) and 4(b)), whereas the contrary results were observed in the GALNT8-suppressed DLD1 and LoVo cell lines (Figures 4(c) and 4(d)). With all these results, it is suggested that GALNT8 contributes to CRC cell proliferation.

To further explore the oncogenic partnership of the GAU1/GALNT8 cluster in CRC, siGALNT8 or negative control was transfected into GAU1-overexpressing cell lines to examine whether GALNT8 silence could rescue GAU1 overexpression-mediated enhanced proliferation of CRC.
The CCK-8 and colony formation assay results demonstrated that the upregulated cell proliferation and colony formation in the GAU1-overexpressed SW620 and HCT116 cell lines were partially attenuated by siGALNT8 in Figures 4(e) and 4(f)), suggesting that GALNT8 is a critical downstream operator of GAU1 during the CRC proliferation.

3.5. Overexpression of GAU1/GALNT8 Axis Sensitizes CRC Cell Lines to Chemotherapy. Given the experimental evidence that GAU1/GALNT8 axis overexpression significantly promotes the cancer cell proliferation and GAU1 boosts cell cycle by increasing S-phase entry, we further questioned if GAU1/GALNT8 axis can reshape the drug response of cancer cells to chemotherapy agents targeting DNA replication. The in vitro oxaliplatin drug response data revealed that cancer cells overexpressing GAU1 or GALNT8 are more vulnerable to chemotherapy agents causing replication fork collapse, indicating GAU1/GALNT8 axis as a potential actionable target for the personalized medicine of CRC. This finding was 
further confirmed by the drug resistance phenotype in GAU1/GALNT8 knockdown cell lines (Figure 4(g)).

\section{Discussion}

CRC is one of the most common and lethal types of cancer [16]. In the past decades, genetic alteration including APC and $K-R A S$ somatic mutation has been identified to cause $70 \%$ of the CRC cases [17] and widely adapted into the diagnosis and drug response prediction during CRC management [18].

Recent studies attributed the transcriptional alteration of the lncRNAs as a hallmark of tumor development $[19,20]$. The enormous efforts on the landscaping of lncRNA expression in cancer $[21,22]$ led to a number of fabulous investigations that improved the understanding of multiple major cancer types [7].

In this study, we identified GAU1 as one of the major oncogenic lncRNAs for CRC by mining the ab initial strategy-based lncRNA database MiTranscriptome [10, 11]. According to our analysis, GAU1 ranked one of the most differentially expressed lncRNAs between CRCs and normal tissues/cell lines (99.75\% percentile of SSEA). Moreover, the overexpression of GAU1 leads to a significant reduction in CRC patient survival $\left(P=3.04 \times 10^{-2}\right)$. After experimentally validating the procancerous ability of GAU1 by the cell proliferation assay after GAU1 expression manipulation in CRC cell lines, we further located GALNT8 as the mostly coexpressed protein-coding gene for GAU1.

GALNT8 encodes a 637-amino-acid type-II membrane protein (GalNAc-T8) [23]. The protein is a member of the UDP-GalNAc polypeptide $\mathrm{N}$-acetylgalactosaminyl transferase (ppGaNTase) family, which initiates mucin-like O-linked protein glycosylation in the Golgi apparatus [24]. Previous research revealed that GALNT8 is expressed in the heart, placenta, skeletal muscle, liver, and kidney and plays a key role during embryonic development [23]. However, the oncogenic effect of GALNT8 is less characterized. Chai et al. reported GALNT8 as the oncogene in retinoblastoma that potentially drives the cancer development and progression [25] by directly binding to the GALNT8 promoter and boost the transcription of GALNT8 through TCEA1 (Transcription Elongation Factor A1) recruitment, which mechanistically endorsed our experimental data in CRC.

Like GAU1, GALNT8 is also associated with poor CRC prognosis $\left(P=0.31 \times 10^{-2}\right)$. Together with the experimental evidence (1) overexpression or silencing GALNT8 mimicked the cancer cell line phenotypic alteration after GAU1 overexpression or knockout. (2) GALNT8 knockdown attenuated the GAU1 overexpression-induced cell proliferation, and not vice versa; we confirmed GALNT8 as the downstream operator of GAU1 in CRC.

Aside from the surgical operation, systemic chemotherapy with folinic acid, fluorouracil, and oxaliplatin (FOLFOX) is also a main treatment solution for CRC. Our result showed an oxaliplatin hypersensitivity in cancer cell lines overexpressing GAU1/GALNT8. This double-edge sword effect of GAU1/GALNT8 overexpression suggested the GAU1/GALNT8 axis as a potential marker in the precision medicine of CRC, although more experimental evidence should be investigated in the future.

One limitation of this study is we did not provide the molecular interaction between GAU1 and GALNT8. Although we have confirmed GALNT8 as the essential operator for the oncogenic ability of GAU1, further investigation on the regulatory mechanism between these bidirectionally transcribed lncRNA/protein-coding gene pairs needs to be clarified by protein-RNA interaction or DNA-RNA binding assay. According to the previous report that GAU1 and GALNT8 share a cisregulation relationship in retinoblastoma [25] and the mutual promoter region of the two genes, investigation on the mechanism behind the abnormal promoter activation in CRC should be conducted in our future studies.

To our best knowledge, this is the first study systematically reporting the oncogenic cascade of GAU1/GALNT8 axis in CRC. By integrating the differential expression data from 7,256 curated RNA-Seq libraries in MiTranscriptome and experimental validation, we demonstrated that GAU1, together with its downstream protein GALNT8, is associated with cancer cell proliferation, poor patient survival, and chemotherapy response.

\section{Data Availability}

The data used to support the findings of this study are included within the article.

\section{Conflicts of Interest}

The author(s) declare(s) that they have no conflicts of interest.

\section{Authors' Contributions}

Xuemei Tang and Haoyu Ruan contributed equally to this article.

\section{Acknowledgments}

This study was supported by the National Natural Science Foundation of China (81871728 and 81672105 to M.G., 81802993 to Z.W.) and Innovation Group Project of Shanghai Municipal Health Commission (2019CXJQ03 to M.G.).

\section{References}

[1] R. L. Siegel, K. D. Miller, and A. Jemal, "Cancer statistics, 2020," CA: a Cancer Journal for Clinicians, vol. 70, no. 1, pp. 7-30, 2020.

[2] F. Bray, J. Ferlay, I. Soerjomataram, R. L. Siegel, L. A. Torre, and A. Jemal, "Global cancer statistics 2018: GLOBOCAN estimates of incidence and mortality worldwide for 36 cancers in 185 countries," CA: a Cancer Journal for Clinicians, vol. 68, no. 6, pp. 394-424, 2018.

[3] M. J. Schell, M. Yang, J. K. Teer et al., "A multigene mutation classification of 468 colorectal cancers reveals a prognostic role for_APC_," Nature Communications, vol. 7, no. 1, pp. 1174311743, 2016. 
[4] R. Schulz-Heddergott, N. Stark, S. J. Edmunds et al., “Therapeutic Ablation of Gain-of-Function Mutant p53 in Colorectal Cancer Inhibits Stat3-Mediated Tumor Growth and Invasion," Cancer Cell, vol. 34, no. 2, pp. 298-314.e7, 2018.

[5] W. Liao, M. J. Overman, A. T. Boutin et al., "KRAS-IRF2 Axis Drives Immune Suppression and Immune Therapy Resistance in Colorectal Cancer," Cancer Cell, vol. 35, no. 4, pp. 559572.e7, 2019.

[6] R. P. Kuiper, M. J. L. Ligtenberg, N. Hoogerbrugge, and A. Geurts van Kessel, "Germline copy number variation and cancer risk," Current Opinion in Genetics \& Development, vol. 20, no. 3, pp. 282-289, 2010.

[7] A. Bhan, M. Soleimani, and S. S. Mandal, "Long noncoding RNA and cancer: a new paradigm," Cancer Research, vol. 77, no. 15, pp. 3965-3981, 2017.

[8] X. Deng, S. Li, F. Kong et al., "Long noncoding RNA PiHL regulates p 53 protein stability through GRWD 1/RPL 11/MDM 2 axis in colorectal cancer," Theranostics, vol. 10, no. 1, pp. 265280, 2020.

[9] X. Deng, H. Ruan, X. Zhang et al., "Long noncoding RNA CCAL transferred from fibroblasts by exosomes promotes chemoresistance of colorectal cancer cells," International Journal of Cancer, vol. 146, no. 6, pp. 1700-1716, 2020.

[10] C. Trapnell, D. G. Hendrickson, M. Sauvageau, L. Goff, J. L. Rinn, and L. Pachter, "Differential analysis of gene regulation at transcript resolution with RNA- seq," Nature Biotechnology, vol. 31, no. 1, pp. 46-53, 2013.

[11] The RGASP Consortium, T. Steijger, J. F. Abril et al., "Assessment of transcript reconstruction methods for RNA-seq," Nature Methods, vol. 10, no. 12, pp. 1177-1184, 2013.

[12] M. K. Iyer, Y. S. Niknafs, R. Malik et al., "The landscape of long noncoding RNAs in the human transcriptome," Nature Genetics, vol. 47, no. 3, pp. 199-208, 2015.

[13] A. Subramanian, P. Tamayo, V. K. Mootha et al., "Gene set enrichment analysis: a knowledge-based approach for interpreting genome-wide expression profiles," Proceedings of the National Academy of Sciences of the United States of America, vol. 102, no. 43, pp. 15545-15550, 2005.

[14] M. B. Amin, F. L. Greene, S. B. Edge et al., "The eighth edition AJCC cancer staging manual: continuing to build a bridge from a population-based to a more "personalized" approach to cancer staging," CA: a Cancer Journal for Clinicians, vol. 67, no. 2, pp. 93-99, 2017.

[15] Y. H. Xing, R. W. Yao, Y. Zhang et al., “_SLERT_ Regulates DDX21 Rings Associated with Pol I Transcription," Cell, vol. 169, no. 4, pp. 664-678.e16, 2017.

[16] N. Gupta, S. S. Kupfer, and A. M. Davis, "Colorectal cancer screening," JAMA, vol. 321, no. 20, pp. 2022-2023, 2019.

[17] A. L. Masson, B. A. Talseth-Palmer, T.-J. Evans et al., "Copy number variants associated with 18p11.32, _DCC_ and the promoter 1B region of _APC_ in colorectal polyposis patients," Meta Gene, vol. 7, pp. 95-104, 2016.

[18] D. Han, M. Wang, N. Ma, Y. Xu, Y. Jiang, and X. Gao, "Long noncoding RNAs: novel players in colorectal cancer," Cancer Letters, vol. 361, no. 1, pp. 13-21, 2015.

[19] H. Yari, L. Jin, L. Teng et al., "LncRNA REG1CP promotes tumorigenesis through an enhancer complex to recruit FANCJ helicase for REG3A transcription," Nature Communications, vol. 10, no. 1, p. 5334, 2019.
[20] N. Li, G. Yang, L. Luo et al., "IncRNATHAP9-AS1Promotes pancreatic ductal adenocarcinoma growth and leads to a poor clinical outcome via sponging miR-484 and interacting with YAP," Clinical Cancer Research: An Official Journal of the American Association for Cancer Research, vol. 26, no. 7, pp. 1736-1748, 2020.

[21] J. T. Hua, S. Chen, and H. H. He, "Landscape of noncoding RNA in prostate cancer," Trends in Genetics, vol. 35, no. 11, pp. 840-851, 2019.

[22] M. Klingenberg, A. Matsuda, S. Diederichs, and T. Patel, "Non-coding RNA in hepatocellular carcinoma: mechanisms, biomarkers and therapeutic targets," Journal of Hepatology, vol. 67, no. 3, pp. 603-618, 2017.

[23] K. E. White, B. Lorenz, W. E. Evans, T. Meitinger, T. M. Strom, and M. J. Econs, "Molecular cloning of a novel human UDP-GalNAc: polypeptide n-acetylgalactosaminyltransferase, GalNAc-t8, and analysis as a candidate autosomal dominant hypophosphatemic rickets (ADHR) gene," Gene, vol. 246, no. 1-2, pp. 347-356, 2000.

[24] K. ten Hagen, T. A. Fritz, and L. A. Tabak, "All in the family: the UDP-GalNAc: polypeptide N-acetylgalactosaminyltransferases," Glycobiology, vol. 13, no. 1, pp. 1r-16, 2002.

[25] P. Chai, R. Jia, R. Jia et al., "Dynamic chromosomal tuning of a novel GAU1 lncing driver at chr12p13.32 accelerates tumorigenesis," Nucleic Acids Research, vol. 46, no. 12, pp. 60416056, 2018. 